\title{
Le rêve et la mort dans les Oneirokritika d'Artémidore ${ }^{1}$
}

INTRODUCTION

Malgré des différences évidentes, le rêve et la mort présentent, en tant que constantes fondamentales de l'humanité, quelques rapports et points communs ${ }^{2}$ :

a) Tous deux, le rêve comme la mort, échappent entièrement à la perception humaine. On ne peut pas fabriquer son rêve, ni le produire, et en aucun cas il n'est possible d'en influencer le contenu et la symbolique. Il existe par lui-même. Nous ne savons pas non plus quand il nous en arrivera un que nous pourrons aussi garder en mémoire. Notre mort, nous pouvons certes la décider, donc la causer, mais il est normalement tout à fait impossible de déterminer le moment où elle surviendra ${ }^{3}$. Le fait est en soi inévitable. Plus que tout, cependant, un point est essentiel : nul ne sait de façon explicite ce qui se passe au moment de mourir ni ce qui s'ensuit, ainsi que ce à quoi le mort doit s'attendre.

b) Tous deux constituent un état ou un univers qui est clairement distinct du temps de vie de l'homme, mais a cependant une présence immédiate et

1. Le texte qui suit constitue la version retravaillée de ma communication de Montpellier. Je remercie les membres du " Groupe Artémidore " pour les suggestions faites lors de la discussion, et avant tout Julien du Bouchet et Christophe Chandezon pour leur invitation et leur hospitalité, et pour la traduction de mon texte. Mes remerciements pour les aides diverses apportées lors de la mise au point de mon manuscrit vont à Monika Duldner, Christopher Schliephake, Johann Schmalhofer et Johannes Sedlmeyr. Toutes les citations d'Artémidore renvoient à PACK 1963.

2. Approche anthropologique et sociologique de ce de thème : HumpHreys 1981 ; Hopkins 1983 ; MACHO 1987 ; Assmann 2002 ; WALDE, ENGLHOFer et BinDER 2002. Dans une perspective psychologique: vON Franz 2001.

3. Pour une définition globale des différentes conceptions de ce qu'est la mort : BAUdy 2001. Sur les limites de la connaissance: MACHO 2000. 
effective dans ce temps de vie. Le rêve peut influencer l'homme dans son action, que celui-ci en ait conscience ou non, en l'effrayant ou en le rassurant. La mort peut pousser un homme à s'interroger sur ce qui lui reste à vivre et sur la manière dont il doit organiser sa vie en fonction de ce qu'il croit devoir attendre de ce qu'il y a après la mort. En dernière analyse, c'est la mort qui structure la vie.

c) Dans les deux états, le rêve et la mort, l'homme n'est plus maitre de luimême et ne dispose donc plus de son libre arbitre. Ce n'est donc pas sans raison que, depuis l'Antiquité, le sommeil - qui est un préalable indispensable au rêve - passe pour un état très proche de la mort ${ }^{4}$.

d) Tous deux, en fin de compte, entretiennent des rapports particulièrement étroits, car dans de nombreuses civilisations on attribue aux mourants une activité onirique plus intense ${ }^{5}$.

En outre, dans l'Antiquité gréco-romaine, le lien entre le rêve et la mort se traduit par une grande quantité d'exemples historiques. C'est ainsi que la mort des empereurs romains (ou déjà celle de Jules César) est annoncée en rêve, aussi bien au protagoniste lui-même qu'à d'autres personnes. C'est ainsi également qu'on procède au décompte posthume des rêves les plus affreux possible. Les plus éminents historiens les ont transmis et il ressort de cela que leurs lecteurs considèrent comme plausible que les rêves indiquent une mort imminente ${ }^{6}$. Aussi devons-nous présupposer que la mort est également présente dans les rêves des hommes ordinaires qu'Artémidore a recueillis et interprétés dans son œuvre.

Le matériau artémidorien se laisse classer en deux catégories. Dans la première, on visualise différents aspects de la mort, dans la mesure où l'on rêve de sa propre mort, d'autres personnes mortes, des monuments funéraires?

4. À propos du sommeil, qui suscite depuis peu l'intérêt des chercheurs : WÖHRLE 1995 ; WIEDEMANN 2003.

5. Voir à ce sujet Habermehl 1992 ; von Franz 2001, p. 241-242; Harris 2009, p. 25 (sur Platon, Criton, 44a-b). Alt 2002, p. 46, fait remarquer pour Artémidore que faire un rêve quand on est face à la mort est " aussi un acte diététique de purification ".

6. La documentation est rassemblée et interprétée dans WeBER 2000, p. 417-496, avec renvoi à la bibliographie.

7. Un rêve de ce genre (II 61) est ensuite interprété en fonction du statut social, par exemple comme positif pour les esclaves, qui ne peuvent normalement pas posséder de monument funéraire (Ariès 1984, p. 10-11). Pour la signification des monuments en eux-mêmes : von Hesberg et ZaNKer 1987, p. 17-18. 
des funérailles, ou bien le monde des morts et les dieux qui y règnent ${ }^{8}$. Mais les rêves de ce genre n'annoncent en aucun cas nécessairement la mort. Cela vaut au premier chef pour le traitement de nombreux aspects de la symbolique de la mort à la fin du livre II, traitement qui n'occupe pas moins de quatorze chapitres. C'est ainsi qu'Artémidore a composé tout un chapitre sur un seul aspect de la question : "Rêver qu'on meurt, qu'on est conduit au cimetière et enterré. " Ce rêve connaît toute une série d'interprétations différentes, à savoir pour un esclave avec ou sans poste de confiance, pour une personne mariée ou non, pour une personne vivant dans son pays ou à l'étranger, pour des athlètes, des lettrés, des pères de famille, pour quelqu'un qui a peur de quelque chose, pour ceux qui veulent faire des opérations foncières, pour des gens en procès et pour un malade.

À la seconde catégorie appartiennent des rêves au contenu varié dispersés dans l'ensemble de l'œuvre, qu'Artémidore interprète comme indications d'une mort imminente. Dans ce contexte, il est remarquable que, sur les 95 exemples de rêves du livre $\mathrm{V}$, presque la moitié, 40 pour être précis, renvoient à la mort ${ }^{10}$. D'un point de vue purement quantitatif, les rêves et les interprétations qui se rapportent à la mort occupent une place considérable. Cette constatation prouve à elle seule la signification et la perception particulièrement importantes de la mort dans les Oneirokritika.

À la question de savoir ce que l'on peut retirer, pour comprendre la mort et l'au-delà, des rêves, de leurs interprétations et des justifications de celles$\mathrm{ci}$, je souhaiterais répondre en quatre points ${ }^{11}$ :

1. Comment les différents aspects de la mort sont-ils visualisés dans les rêves, et qui sont les gens qui rêvent de la mort le plus souvent ?

2. Quel est le statut qui revient au mort lui-même? Comment s'occupet-on du corps?

3. Quelles sont les conceptions de l'au-delà que livre ce matériau?

8. Sur les dieux du monde souterrain (II, 39) : SchwabL 2004, p. 22-23.

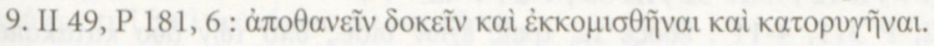

10. Les livres I-IV d'Artémidore ne comportent pas moins de 127 rêves dont l'interprétation les rapporte à la mort.

11. À propos de l'utilité fondamentale d'Artémidore précisément aussi pour ce genre de questionnement : BöHME 2008, p. 25-26. À propos des méthodes d'interprétation: WEBER 1999, p. 215-219; WALDE 2001, p. 171-222. 
4. De quelle manière la mort - qui englobe bien plus que la simple condition mortelle de l'homme - détermine-t-elle la vie des hommes, dans une perspective à la fois émotionnelle et rationnelle?

Il s'agira donc, d'un côté, de pratiques concrètes, de l'autre, d'attitudes mentales relatives à la mort et de représentations que l'on en avait.

\section{LES DIFFÉRENTS ASPECTS DE LA MORT VUS EN RÊVE}

ET LES TYPES DE PERSONNES

QUI RÊVENT DE LA MORT LE PLUS SOUVENT

Il apparaît que les visions oniriques présentent un grand nombre de façons de mourir, allant de la décapitation et avant tout de la crucifixion - qui, à l'origine, n'était pas pratiquée dans le monde grec et, à l'époque d'Artémidore, s'appliquait en priorité aux esclaves ${ }^{12}$-, de la mort par pendaison, dans un incendie ou à l'arme blanche à la mort sous le coup de la foudre, à celle reçue d'animaux sauvages comme les éléphants ou les loups, au fait d'être enterré vivant ou même à l'égorgement ${ }^{13}$. Un exemple :

Un homme, qui avait trois fils, rêva que par deux d'entre eux il était coupé en morceaux et mangé, mais que le plus jeune, se tenant auprès, était en colère contre ses frères et en chagrin, et que, abominant la chose, il disait : " Moi, je ne mangerai pas de mon père. " Il arriva que son plus jeune fils mourut : seul en effet, étant mort avant son père et n'en ayant pas hérité, il ne devait pas manger, je ne dis pas des chairs, mais des biens de fortune. Les deux autres, qui avaient mangé, devinrent les héritiers de la fortune du père ${ }^{14}$.

À côté de la mort dans ces images plutôt extraordinaires - la peine de mort en tant que telle n'est pas perçue comme un problème -, il y a en rêve des cas de mort très ordinaire et «normale ». Ils se rapportent soit au rêveur lui-même, ce qui est très fréquent, soit à des membres de sa famille

12. II 53 et IV 49, voir NP, III, p. 225-226, s. v. "Crux » [G. Schiemann].

13. I 2 ; II 9 ; II 12 ; II $49-52$; III 66 ; V 2 ; V 42.

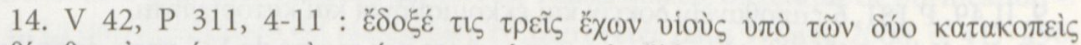

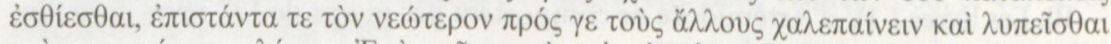

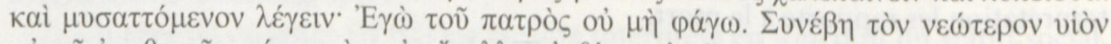

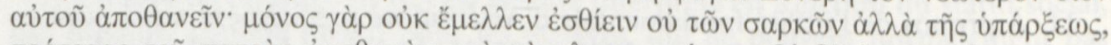

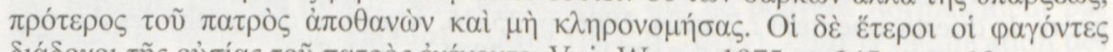

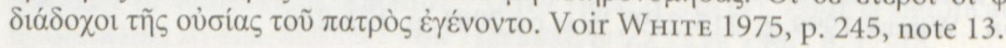


ou à des personnes de son entourage, comme par exemple des compagnons de servitude ${ }^{15}$. En d'autres termes, le rêveur se voit lui-même mourir ou bien il voit périr d'autres personnes de diverses manières. Soulignons ici une constatation digne d'intérêt : il y a certes des visions oniriques du sui$\operatorname{cide}^{16}$, mais jamais le rêveur ne tue quelqu'un d'autre en rêve et ne devient, par là, un meurtrier. Cela doit être considéré moins comme le résultat d'un tabou $^{17}$ qui aurait empêché de rapporter un rêve de ce genre que comme la peur concrète que ce rêve, une fois raconté, puisse se réaliser et que l'on en vienne à être soi-même soupçonné de meurtre.

Dans les rêves agit une foule de divinités et surtout de morts, qui peuvent être connus ou inconnus du rêveur. Par exemple, en rêve, "lutter avec un mort indique maladie ou dispute avec l'un des descendants ou héritiers du mort ${ }^{18} »$. On rencontre aussi - il faudra y revenir - la vision de la descente dans le monde souterrain, dans l'Hadès, et celle où l'on en remonte, ainsi que la vision de divers aspects des funérailles. Dans un passage, lors de l'interprétation d'un rêve, apparaît aussi le mythe de la Gorgone ; à cette occasion, Artémidore tend à adopter une interprétation négative, mais sans pousser très loin, puisqu'il considère ce rêve comme absurde ${ }^{19}$.

Dans ces rêves, comme dans leur interprétation, il est souvent question de la mort de proches ${ }^{20}$. Les interprétations ne se rapportent que rarement à des réactions émotionnelles, mais plutôt à des questions économiques, ce que les deux exemples choisis ont déjà montré : des thèmes comme ceux des relations entre les générations ou de la transmission du patrimoine, à propos desquels il est aussi question de la prise en charge des parents par les enfants, étaient donc toujours d'une grande importance.

15. I 2 ; I 15; I 79; II 49; IV 64; V 2 ; V 42 ; V 61. Pour le contexte et des renvois à d'autres auteurs: HARRIS 2009, p. 59 et suiv.

16. Portmann 1993, p. 235-236; VAn Hooff 2006.

17. À propos des tabous relatifs à la mort: WALDE, ENGLHOFER et BINDER 2002, p. 642-643.

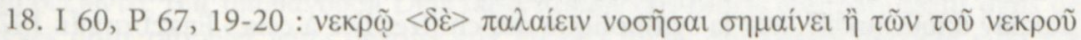

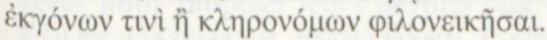

19. IV 63, P 287, 1-7. Voir Wiegels 1991, p. 23-25, qui considère que l'image de la Gorgone personnifie l'anéantissement de toute vie.

20. Chez Artémidore, il n'est à vrai dire pas question d'entrer en communication avec des morts (réels) par le moyen du rêve; il en va différemment dans la culture juive, voir HASAN-ROKEM 1999. 
La plupart des rêveurs auxquels des visions oniriques annoncent la mort sont des malades. Artémidore ne dit presque rien de leurs maladies, de sorte qu'il est difficile de s'en faire une idée et de préciser s'il s'agissait seulement de personnes gravement malades. Pour celles-ci, néanmoins - indépendamment de l'appréciation des capacités qu'avait alors la médecine, la probabilité que survienne la mort était extrême ; l'attention accrue portée aux rêves des malades est due aussi à la proximité de la mort et, partant de là, à une réceptivité particulière à l'égard des rêves. Artémidore formule cela de manière explicite à propos du rêve dans lequel on quitte son propre corps comme un serpent quitte sa peau, ce qui indiquait une mort imminente: "C'est que l'âme, sur le point de quitter le corps, lui mettait en tête de telles imaginations ${ }^{21}$. "

La mort accompagnait constamment le voyageur ${ }^{22}$ et celui qui était en procès ; elle était présente lors des maladies ou bien à l'issue d'une vieillesse vénérable qui était ressentie comme un poids et une peine et pour laquelle il n'existait aucune prise en charge organisée par la communauté. Lorsqu'un malade rêve d'une vieille femme, cela indique la mort, car « une vieille est par elle-même symbole de conduite au cimetière, puisqu'elle doit mourir dans peu de temps $s^{23}$ ". Certes, il y a les visions oniriques de la rubrique « guerre et actes des guerre ", qui annonçaient soit la mort aux gens âgés et aux malades, soit l'éphébie, mais la mort à la guerre ne joue plus aucun rôle dans ces visions. Cela ne doit pas étonner, car les cités d'Asie Mineure ne se faisaient plus la guerre entre elles à cette époque. À la rigueur, ce genre de mort pouvait survenir lors du service militaire pour les membres de l'armée romaine.

Dans ce contexte, il faut ajouter que les rêves qui contiennent la mort sont très largement dépassés en nombre et en variété par les rêves dont les visions n'ont certes aucun lien avec la mort, mais sont interprétées comme indiquant la mort ${ }^{24}$. Par exemple, " quand les fourmis se promènent sur le

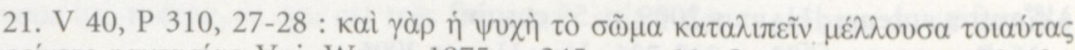

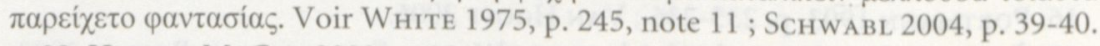

22. Harris-McCoy 2009, p. 98-100.

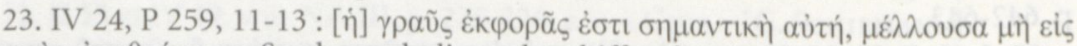

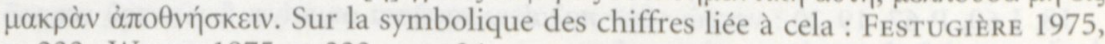
p. 233 ; White 1975 , p. 223 , note 24.

24. I 4 ; I 5 ; I 13 ; I 14 ; I 16 ; I 21 ; I 24 ; I 26 ; I 27 ; I 30 ; I 31 ; I 33 ; I 35 ; I 39-44 ; I 47 ; I 48 ; I 50 ; I 51 ; I 54 ; I 56 ; I $58-60$; I 63 ; I 66 ; I 67 ; I 70 ; I 76-80 ; I 82 ; II 2 ; II 3 ; II 7 ; II 9 ; II 10 ; II $12-23$; II $29-31$; II 33 ; II $36-39$; II 45 ; II 68 ; III 5 ; III 6 ; III 28 ; III 30 ; III 32 ; III 36 ; III 41 ; III 46 ; III 49 ; III 50 ; III 53 ; III 56-58; IV 4 ; IV 19; IV 24; IV 27 ; IV 29; IV 30 ; IV $32-34$; IV 36 ; IV 39 ; IV 40 ; IV 43 ; IV 47-49; 
corps du songeur, elles prédisent mort, parce qu'elles sont filles de la terre, et de sang froid, et noires ${ }^{25}$ ". Ici aussi, les rêves de malades forment de loin l'essentiel de la matière.

\section{LE STATUT DU MORT ET LA MANIÈRE DE S'OCCUPER DU CORPS}

Tout d'abord, il apparaît grâce à divers passages d'Artémidore que le monde des vivants était nettement séparé du monde des morts ${ }^{26}$. À la mort est lié un changement d'état évident, une altérité radicale, parce que "le mort ne reste pas au même lieu ${ }^{27}$ "; ou bien encore " les mendiants ont analogie avec la mort, du fait que seuls de tous, comme la mort, s'ils ont reçu, ils ne rendent rien $^{28}$ ". Ce qui veut dire que tout ce qui est tombé du côté de la mort y reste. C'est pourquoi il est négatif, voire dangereux, que des morts reviennent à la vie et agissent - même si ce n'est qu'en rêve -, " car les morts n'ont plus commerce avec les vivants, et non plus en retour les vivants avec les morts ${ }^{29}$ ". C'est pour cette raison que le seul fait d'être touché par des morts en rêve est considéré comme dangereux. Le rêve dans lequel des morts reviennent à la vie n'apporte qu'ennuis et préjudices, et ce de manière tout à fait terre-à-terre : "Qu'on se figure en effet, par hypothèse, quel trouble il y aura si des morts ont ressuscité. Outre cela, comme

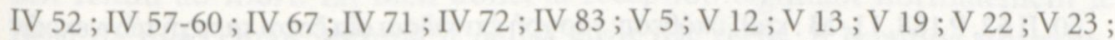
V $25-27$; V 30 ; V 31 ; V 33-35; V 37;V 39-41; V 43 ; V 46 ; V 49 ; V 50 ; V 52 ; $\mathrm{V} 59 ; \mathrm{V} 60 ; \mathrm{V} 62 ; \mathrm{V} 63 ; \mathrm{V} 71 ; \mathrm{V} 73 ; \mathrm{V} 82 ; \mathrm{V} 84 ; \mathrm{V} 85 ; \mathrm{V} 88 ; \mathrm{V} 92-94$.

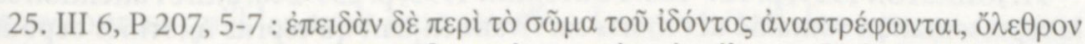

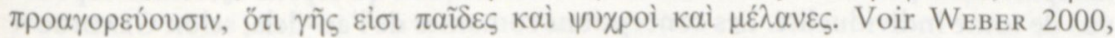
p. 450 , note 211 .

26. Sur le concept des deux sphères (l'endosphère et l'exosphère) : VEIT 2008, p. 23-26, et, en outre, WIEgels 1991, p. 8-9; HoHeISEL 1993, p. 324-325.

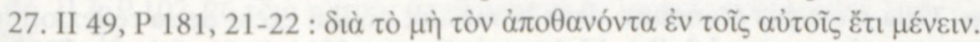

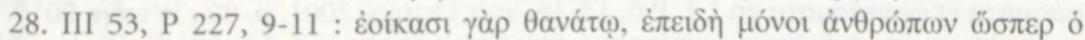

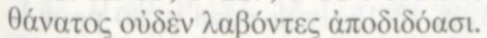

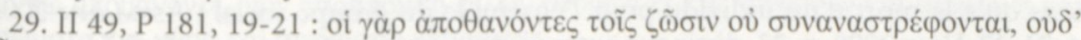

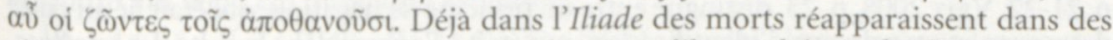
rêves pour rappeler leurs devoirs aux vivants qu'ils ont laissés derrière eux, voir Colpe, Dassmann, Engelmann, Habermehl et Hoheisel 1996, p. 259. L'apparition en rêve de défunts peut aussi être considérée comme une preuve que l'âme d'un mort n'a pas disparu avec la vie, voir RoHDE 1925, vol. II, p. 392-393. Sur la pratique de la nécromancie et de la nékyomancie, qui est réprouvée par Artémidore (II 69) : OGDEN 2001, en particulier p. 75-92 sur la signification des rêves ; BREMMER 2002, p. 71-83. 
il est naturel, ils réclameront aussi leurs biens, d'ou résulteront aussi des dommages ${ }^{30}$. " Nous connaissons déjà la perspective matérielle qui est ici adoptée. Mais on supposait aussi que les âmes de ceux qui n'avaient pas fait l'objet des rites funéraires qui leur étaient dus ou qui avaient connu une mort précoce et malheureuse revenaient pour se venger des responsables et pour infliger des souffrances aux envieux ${ }^{31}$.

Les rites étaient donc importants et concernaient aussi bien les morts eux-mêmes que les groupes sociaux souillés par la mort auxquels le défunt appartenait ${ }^{32}$ : le traitement usuel appliqué à un mort comportait l'exposition du corps en bonne et due forme, puis l'enterrement. La pratique normale chez Artémidore est l'inhumation : elle était depuis longtemps usuelle dans l'aire culturelle grecque, et s'est aussi imposée au $\mathrm{II}^{\mathrm{e}}$ siècle de notre ère dans les provinces occidentales et septentrionales de l'Empire romain ${ }^{33}$. Cela est du reste un indice supplémentaire de l'ancrage du traité dans l'Orient romain, puisqu'il n'y est jamais question de crémation. Le rêve de la découverte d'un trésor annonce dommages et tracas, souvent même la mort : il est « impossible en effet de trouver un trésor sans excavations, comme il est impossible de déposer un mort sans qu'on ait creusé la terre ${ }^{34}$ ». Quand Artémidore explique que « les morts sont enveloppés de haillons déchirés

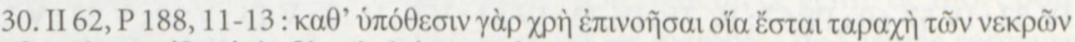

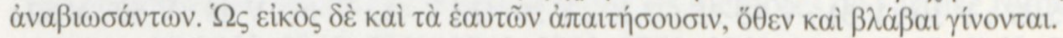

31. Johnston 1999, p. 127-160. Portmann 1993, p. 239-240, attire l'attention sur la représentation romaine des morts survivant en tant qu'esprits informes dépourvus de toute individualité. Les conceptions romaines de l'au-delà, selon GLADIGow 1980, p. 128-131, n'ont pris forme que durant le Principat, de même chez ColPE, Dassmann, Engelmann, Habermehl et Hoheisel 1996, p. 292-301. Artémidore n'a visiblement pas intégré cette conception, ignorant même les rêves terrifiants de fantômes. Cela n'est pas étonnant, dans la mesure où il a exclu de sa réflexion les

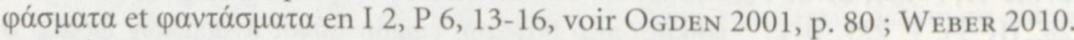

32. À propos des rites usuels: Wiegels 1991, p. 10-15; voir aussi p. 20 pour le sens qu'a la mort d'un individu pour l'ensemble du groupe social ; Assmann 2002, p. 21-22. VeIT 2008, p. 23, distingue entre un principe de négation, qui était lié au culte des morts, et un principe d'identification, qui était à l'œuvre dans le culte des ancêtres.

33. Voir ToynbeE 1971, p. 39-42. Voir en outre l'analyse de la question par FABER, FASOlD, STRUCK et WitTEYer 2007, p. 11-16, où l'on a également SCHEID 2007, qui ne s'occupe pas d'Artémidore, mais montre clairement que ce changement n'avait pas de motif religieux.

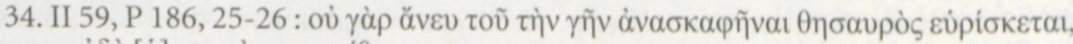

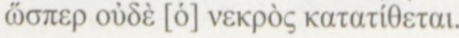


comme les nourrissons et on les pose à terre ${ }^{35}$ ", il y a alors une contradiction certaine, peut-être liée au statut social, avec l'affirmation selon laquelle « les morts sont emportés au cimetière dans les vêtements blancs ${ }^{36} »$. Lorsque quelqu'un n'était pas enterré dans un habit blanc, c'était un signe de pauvreté. Un autre détail nous est donné : on ne coupait pas les cheveux du mort. De l'exposition du corps rien n'est dit, mais il est question du cortège funèbre, du sacrifice et du banquet funéraire. Artémidore fait en effet le parallèle entre le mariage et la mort : " car il leur arrive à tous deux, et celui qui se marie et celui qui meurt, les mêmes choses, par exemple cortège processionnel d'amis hommes et femmes, couronnes, aromates, parfums, liste écrite des biens ${ }^{37}$ ». Pour ce qui est des couronnes, avec lesquelles on parait aussi bien les morts que les tombeaux, nous apprenons qu'on les confectionnait à partir de plantes qui ne risquaient pas trop de se flétrir et de pourrir, à savoir des sortes d'immortelles et de céleri ; ce dernier était du reste aussi utilisé pour les vainqueurs aux concours isthmiques et néméens. Le mort lui-même recevait un certain nombre d'offrandes ; parmi celles-ci sont évoqués les huiles parfumées, les vêtements, les monnaies et les aliments ${ }^{38}$, et l'on pouvait aussi graver une inscription funéraire ${ }^{39}$. Nous apprenons enfin qu'il était d' " usage pour les compagnons de club [sumbiôtai] d'entrer dans les demeures des compagnons morts et d'y dîner et, dit-on, cette réception est donnée par le mort en reconnaissance de l'honneur que les compagnons ont rendu au mort. Eh bien donc, la réception signifiait normalement le péril de mort, mais comme il ne les avait pas reçus, il échappa normalement au péril. D'autre part, il y eut naufrage, parce que c'est par manque de ressources qu'il les avait repoussés ${ }^{40}$ ». Le mort était donc perçu comme

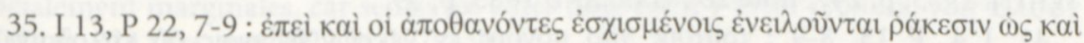

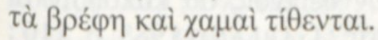

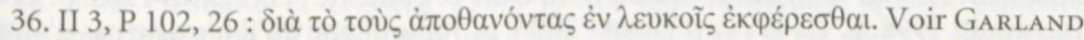
1985 , p. 24.

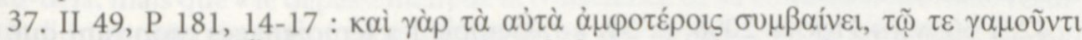

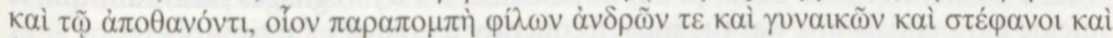

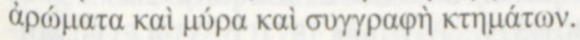

38. I 4 ; I 5 ; II 3 ; II 49 ; IV 2 ; IV 22 ; IV 81 . Sur les aliments pour les morts : Colpe, Dassmann, Engelmann, Habermehl et Hoheisel 1996, p. 259.

39. V 76. Sur la signification des inscriptions funéraires et sur le problème du statut social auquel elles sont liées : Petrucci 1998, p. 20-22.

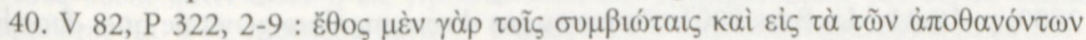

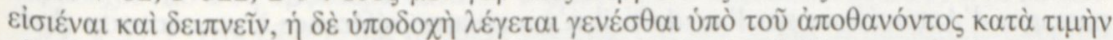

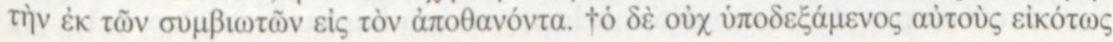


l'hôte qui donnait le banquet. Un autre détail aussi est important à propos du banquet funéraire, d'autant qu'il concerne le suicide qui est souvent évoqué dans les visions oniriques. Ceux qui s'étaient pendus passaient pour déshonorés, idée que l'on trouve aussi chez Artémidore ; ils ne reçoivent pas de funérailles en bonne et due forme et cela se traduit par le fait que " les proches ne les appellent pas par leur nom aux repas funéraires ${ }^{41} »$. Il y a débat parmi les chercheurs pour savoir s'il faut voir dans le jugement en partie positif touchant la pendaison ${ }^{42}$ une conception émanant de cercles philosophiques ou bien s'il y a derrière cela un sentiment général. Les indications que l'on trouve chez Artémidore parlent en faveur de la première solution.

Dansl'ensemble, en ce qui concerne la manière dont on s'occupe du corps, il n'est pas possible de dresser un tableau d'ensemble à partir d'Artémidore, ce qui ne doit pas étonner, étant donné la multiplicité des rites connus par ailleurs. Ses remarques offrent cependant des indications précieuses à propos des normes de comportement et de quelques détails d'importance.

\section{LES CONCEPTIONS DE L'AU-DELÀ DANS LES ONEIROKRITIKA ${ }^{43}$}

Elles constituent l'aspect le plus difficile, car non seulement il y avait en ce domaine un spectre très large de conceptions, mais celles-ci étaient en outre liées entre elles de manière inconsistante et inconséquente. Les quatre concepts grecs les plus importants étaient l'entrée dans un Hadès de déso-

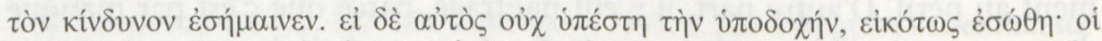

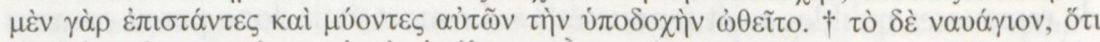

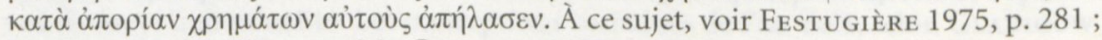
White 1975, p. 247, note 26 ; Garland 1985, p. 39.

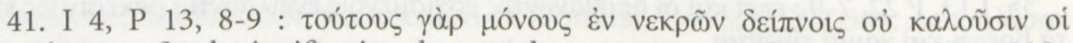
$\pi \rho о \sigma \eta ́ \kappa o v \tau \varepsilon \zeta$. Sur la signification du nom dans ce contexte : GARLAND 1985, p. 110 ; Petrucci 1998, p. 18-19. van Hooff 2006, p. 36, souligne qu'il n'existe pas d'autre attestation de ce fait.

42. I 50. van Hooff 2006, p. 34, a rassemblé les témoignages montrant que le suicide par pendaison "was the most frequently used exit in antiquity ".

43. À ce sujet Rohde 1925, vol. I, p. 301-319; Toynbee 1971, p. 33-42 ; Gladigow 1974 ; Hinard 1987 ; Baltes 1988; Hoheisel 1993; Wiegels 1991 ; Pekary 1994 ; Colpe, Dassmann, Engelmann, Habermehl et Hoheisel 1996 ; NP, V, p. 897-899, s. v. "Jenseitsvorstellungen " [Fr. Graf] ; Portmann 1993, p. 231 ; BREMMER 2002. Sur la diversité des concepts, souvent contradictoires : Albinus 2000, p. 10-11; Le BrIs 2001. Remarquons d'une manière générale que, dans les passages d'Artémidore sur l'au-delà, il n'y a aucune référence à un contexte chrétien, ce qui surprend tout de même quelque peu, voir Samellas 2002, p. 2-3. 
lation, la vie bienheureuse dans l'Élysée ${ }^{44}$, le strict nihilisme des épicuriens à propos de l'au-delà - donc la mort vue comme le néant ${ }^{45}$ - et, enfin, l'expiation dans l'autre monde des méfaits commis dans celui-ci ${ }^{46}$.

Les conceptions sur lesquelles reposent les interprétations d'Artémidore forment des couches extrêmement nombreuses. Il revient plusieurs fois sur ce qui arrive au corps après les funérailles : « de la morte en effet le composé se dissout dans la matière dont il a été formé et composé, et le principal de cette matière, étant du terreux, va rejoindre l'élément qui lui est connatu$\operatorname{rel}^{47}$ ». C'est pour cette raison aussi que le rêve d'avoir un rapport sexuel avec un conjoint défunt indique en général la mort, " car les morts ont changé en terre, et ainsi le fait de les pénétrer n'est rien d'autre que d'être poussé vers la terre et le fait d'être pénétré par eux rien d'autre que d'accueillir de la terre en son corps. Ces deux choses donc signifient mort ${ }^{48}$ ». Pour cette même raison, le cep de vigne est lui aussi un signe de la mort, " parce que la vigne sort de la terre - et c'est dans la terre aussi que se dissolvent les cadavres - et parce que la vigne est spoliée de son fruit au point suprême de sa beautét ${ }^{49}$ ". Toutefois, que l'homme ne puisse être réduit à son corps privé de vie ressort

44. En V 16, P 305, 17-21 (voir Schwabl 2004, p. 25), il est question du rêve d'un

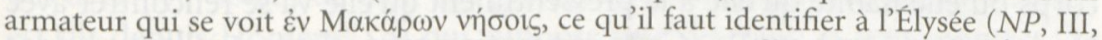
p. 1004-1005, s. v. "Elysion " [Chr. SourvinOu-Inwood]) ; à vrai dire, les Îles des Bienheureux n'existent qu'en rêve, et non en tant que lieu de séjour dans l'au-delà pour l'âme humaine.

45. Sur le scepticisme et l'agnoticisime : Colpe, Dassmann, Engelmann, HABERMeHL et HoHeisel 1996, p. 286-287. La recherche actuelle considère que les représentations de l'au-delà et du salut par les philosophes étaient dans l'ensemble socialement marginales, car seules les élites pouvaient y avoir recours, voir BAUDY 2001, p. 223 ; DAVIES 1999, p. 127-138.

46. Von Hesberg et Zanker 1987, p. 18, font remarquer que la présence constante de la mort ne conduisait pas à une réflexion sur la vie après la mort dans l'au-delà, mais que « le dépassement de la conscience de sa condition mortelle résultait d'un accomplissement plus intense de sa vie, dans lequel la jouissance de son rang social, de ses réalisations et de la reconnaissance était un point essentiel. »

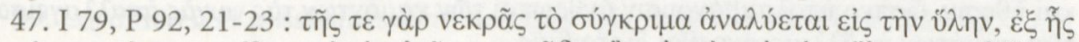

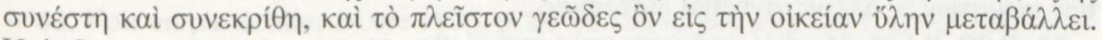
Voir SchWabL 2004, p. 38-39.

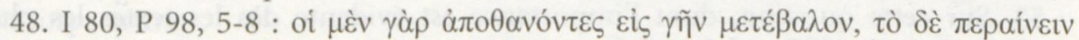

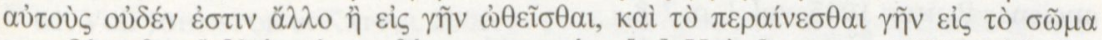

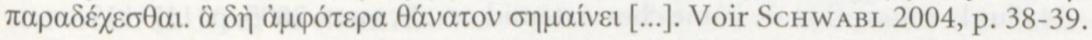

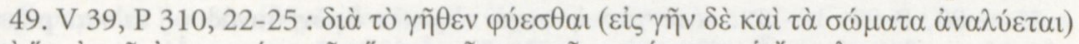

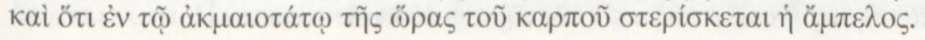


clairement chez Artémidore du fait que la mort sépare l'âme du corps ${ }^{50}$. Cela ressort clairement d'un rêve qui est raconté au livre V :

Un homme rêva que sa sœur avait été séparée de son mari par son père et donnée en mariage à un autre. Il arriva que le songeur mourut. À bon droit le père signifiait le Génie qui pour le songeur était cause de l'existence, et comme la sœur, qui avait même signification que l'âme, avait été séparée du mari, elle devait être, par le Génie, séparée du corps et passer en d'autres lieux de séjour et genres de vie, en la façon où les gens supposent que les âmes des morts, une fois séparées des corps, passent en d'autres lieux de séjour ${ }^{51}$.

Abstraction faite de la question de savoir comment on doit comprendre le daimôn ou génie qui intervient ici, cela ne laisse aucune ambiguïté quant à l'affirmation d'une séparation du corps et de l'âme, comme d'autres passages le confirment. La référenće au dieu Hermès, qui, vu en rêve, annonce la mort pour les malades, vient aussi de croyances traditionnelles, " parce qu'il est considéré comme psychopompe ${ }^{52}$ ». Mais où les âmes sont-elles conduites, et que leur arrive-t-il ${ }^{53}$ ?

Selon Artémidore ${ }^{54}$, l'âme prédit la mort au malade qui rêve qu'il dort avec un dieu ou une déesse, " car c'est quand l'âme est près de quitter le corps où elle habite qu'elle a le pressentiment qu'elle va se rencontrer avec les dieux et s'unir à $e^{55}{ }^{55}$ ». Cette conception est également à l'œuvre dans un autre passage, avec la remarque suivante : « On dit en effet que les âmes délivrées des corps montent au ciel avec une vitesse extraordinaire, et pour

50. Sur les différentes possibilités qu'avaient les Anciens pour faciliter la séparation de l'âme et du corps, voir MorRIs 1996.

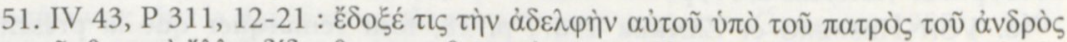

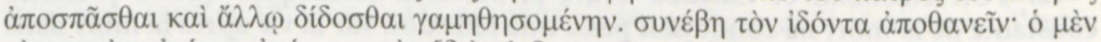

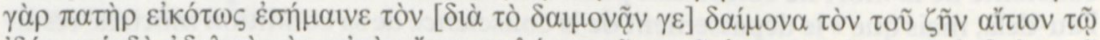

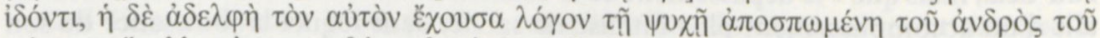

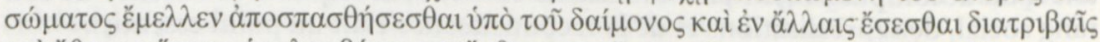

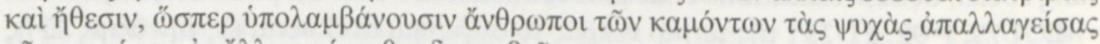

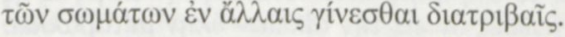

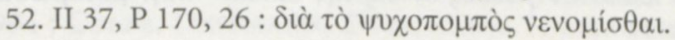

53. Sur cette question, dont l'importance est évidente dès les textes les plus anciens : RoHDE 1925, vol. I, p. 7-8.

54. Sur la conception de l'âme chez Artémidore : Struck 2005, p. 117-119.

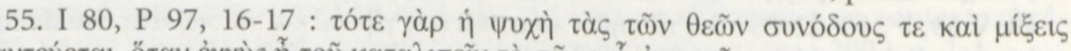

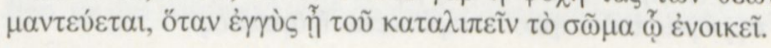


ainsi dire pareilles à des volatiles ${ }^{56}$. " Dans un autre passage encore, Artémidore interprète la montée au ciel comme un signe de mort ${ }^{57}$. Il connaît également l'apothéose, le rituel de divinisation à l'occasion duquel, selon la conception courante, un aigle emportait au ciel l'âme de l'empereur, et l'attribue aux empereurs, aux riches et aux puissants, "car c'est un ancien usage de peindre et sculpter les morts qui sont de cette qualité comme véhiculés sur des aigles et de les honorer par de tels monuments ${ }^{58} »$. Artémidore n'enferme cependant pas le rêve des âmes qui s'envolent au ciel dans des considérations de statut, mais le rapporte d'une manière générale à des malades de toutes sortes. Il ne dit rien, en revanche, de l'endroit précis où se trouvent les âmes, de l'état dans lequel elles sont, ni de ce qu'elles font ${ }^{59}$.

Il est ici instructif de jeter un œil sur le texte grec. Les citations sont toutes introduites par des formulations qui laissent percevoir une certaine

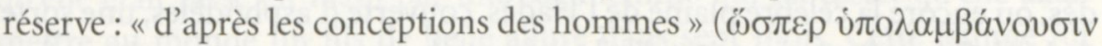

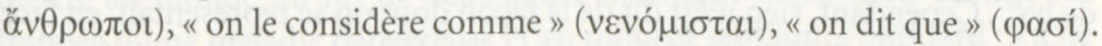
Artémidore a visiblement conscience du fait que de telles conceptions sont des constructions intellectuelles - et cela d'autant plus qu'il travaille selon des méthodes scientifiques et s'appuie avant tout sur l'expérience ${ }^{60}$.

Il ne faut pas s'étonner pour autant que les morts - concrètement les âmes des défunts - soient en bien des passages placés dans l'Hadès, comme cela est dit depuis Homère ${ }^{61}$, ce qui suppose une descente et une montée. On

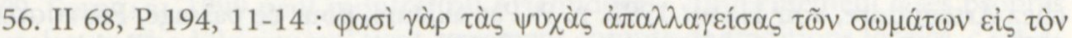

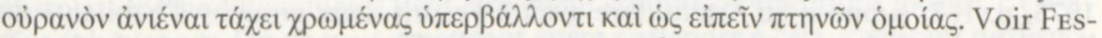
TUGIÈRE 1975, p. 172 ; GARLAND 1985, p. 75, avec les témoignages épigraphiques de cette conception; SCHWABL 2004, p. 39.

57. Sur ce passage (IV 72, P 293, 21-294, 9), où il est question de Plutarque : Habermehl 1992, p. 67, avec des analogies chrétiennes ; White 1975, p. 226, note 51-52; SchWabL 2004, p. 40.

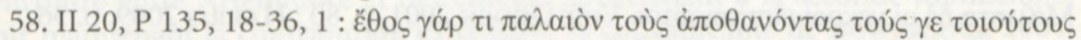

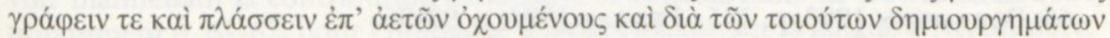

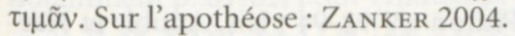

59. Les différentes possibilités sont examinées par HoHeisel 1993, p. 320-321.

60. Voir en particulier Blum 1936, p. 81-91 ; HolowChaK 2001, p. 93-105. HaRRIS 2009 , p. 115, attire l'attention sur d'autres formulations, dont la "vagueness " est surprenante.

61. Voir JohnSTON et KäPPEL 2002, p. 1016 à propos de la topographie du monde souterrain au début du chant XXIV de l'Odyssée d'Homère, où, en autres, est localisé

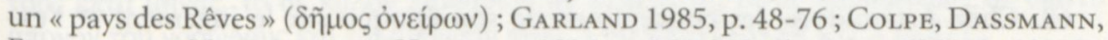
Engelmann, HabermeHl et Hoheisel 1996, p. 262-288; Albinus 2000, p. 13-15. 
trouve des déclarations importantes sur les habitants du monde d'en-bas: "Les habitants de l'Hadès sont inactifs, sans mouvement ", ou encore "Les habitants de l'Hadès n'ont plus de chagrin ni aucun souci' ${ }^{62}$. " Ailleurs il est dit - je cite les passages à la file : «Celui qui est mort est sans maitre et il cesse de peiner et de servir [...] les morts n'ont plus ni crainte ni chagrin [...] les morts n'ont plus ni peine ni maladie ${ }^{63}$. » Cet état de complète absence d'émotions s'exprime aussi dans le fait qu'en rêve les morts sont perçus comme des garants fiables qui disent la vérité en toutes circonstances, car ce sont des êtres " qui ni n'espèrent ni ne craignent rien ${ }^{64}$ ». On a en outre tout l'inventaire du monde souterrain déjà connu par la mythologie ${ }^{65}$ : Pluton et Perséphone, les souverains, auxquels il faut ajouter Isis, Sarapis et Anubis, qui viennent de la tradition égyptienne ${ }^{66}$, mais aussi ses habitants, comme le chien Cerbère, qui en garde l'entrée, Charon, le nocher de l'Hadès, ou encore la célèbre plaine de l'Hadès, couverte d'asphodèles, une sorte de lys ${ }^{67}$. Cependant, rien n'est dit de la façon dont on se représentait, en face de l'absence d'autorité suggérée par le texte, le règne des dieux infernaux qu'évoque Artémidore ${ }^{68}$.

Dans l'ensemble, il est clair que les conceptions de l'au-delà, même au sein d'un seul et même ouvrage comme celui-ci, n'ont rien de cohérent et

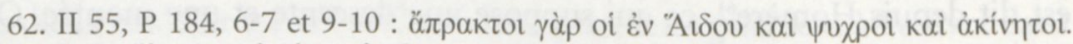

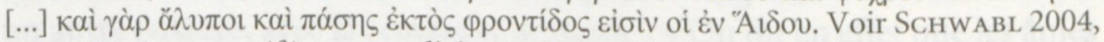
p. 26-27. Aucune référence explicite au Tartare (WIEGELS 1991, p. 15) n'apparaît chez Artémidore, voir Hahn 1992, p. 9. Par ailleurs, pour ce passage, Festugière 1975, p. 167, suppose qu'Artémidore avait à l'esprit deux groupes d'habitants de l'au-delà : les ombres en général et les habitants des Champs Élysées (voir plus haut).

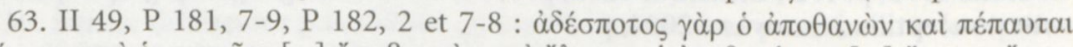

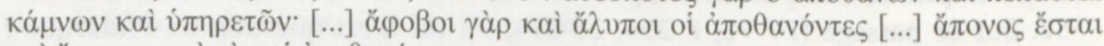

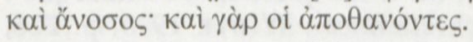

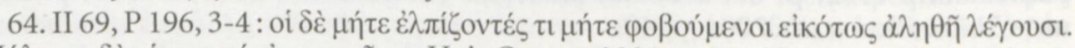

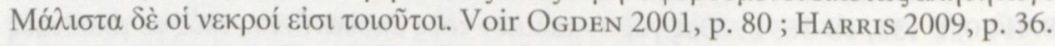

65. Portmann 1993, p. 235 ; Pekary 1994, p. 92-96.

66. Rêves avec Sarapis, qui est identifié à Pluton : V 92-94; Isis, Anubis et Harpocrate : II 34 et 39. Voir White 1975, p. 247, n² 29-30; BArRigón Fuentes 1994 ; Colpe, Dassmann, Engelmann, Habermehl et Hoheisel 1996, p. 317 ; Schwabl 2004, p. 22-23.

67. Sur la prairie d'asphodèles (III 50) d'après Homère, Odyssée, XI, 539 et XXIV, 13 : White 1975, p. 180, note 27 ; Colpe, Dassmann, Engelmann, Habermehl et HoHeISEL 1996, p. 264.

68. Chez Artémidore, les conceptions de son époque sur le jugement et l'expiation dans l'au-delà n'apparaissent pas nettement, alors qu'elles sont sans cesse 
qu'elles se côtoient l'une l'autre sans rapport entre elles ${ }^{69}$. Il est également impossible d'établir aucun lien contraignant entre les rites funéraires usuels et les conceptions de l'au-delà. La mort reste à vrai dire une barrière définitivement insurmontable, ce qui s'exprime par la décomposition du corps rendu à la terre. Ce qu'il advient de l'âme, au contraire, est conceptualisé différemment, dans la mesure où sa remontée depuis l'au-delà est caractérisée de façon tout à fait positive ${ }^{70}$. Le sort qui échoit à l'âme dans l'Hadès est en revanche fondamentalement désespérant : elle est privée de toute émotion et, plus encore, de toute capacité d'action ${ }^{71}$. Pourtant, à travers ses remarques, Artémidore met aussi en évidence le fait que la mort peut parfaitement représenter une délivrance, en fonction de l'histoire personnelle de chacun $^{72}$. Il rapporte ainsi le cas d'une femme malade, qui avait rêvé qu'elle était enceinte, qu'elle ressentait les premières douleurs de l'enfantement et qu'elle était prise en main par une sage-femme qui lui disait qu'elle allait mettre au monde un fils de belle allure dans sept mois. Cette femme fut frappée d'une maladie dangereuse et en mourut sept jours plus tard : « Elle devait en effet déposer le poids et les souffrances après sept mois. D'autre part le beau petit garçon était la mort, qui, parce qu'il met fin aux peines, était préférable à une vie maladive et misérable ${ }^{73}$. » Dans ce contexte, il faut souligner que, chez Artémidore comme dans toute l'Antiquité, la concep-

convoquées par d'autres auteurs du II $^{\mathrm{e}}$ siècle, comme Plutarque ou Lucien : voir Colpe, Dassmann, Engelmann, Habermehl et Hoheisel 1996, p. 309-313.

69. SCHWABL 1999 peut montrer qu'Artémidore - conformément à ses propres postulats méthodologiques - tient largement compte de traditions locales, c'est-àdire éphésiennes. On ne peut cependant guère s'attendre à cela, s'agissant de dispositions mentales. Le BRIS 2001, p. 180, met en évidence des innovations radicales dans les conceptions de l'au-delà, mais montre aussi une grande permanence des traditions antérieures, surtout dans la masse de la population (COLPE, DASSMANN, Engelmann, Habermenl et Hoheisel 1996, p. 287-288). À propos des points de rencontre entre Artémidore et des auteurs appartenant à la seconde sophistique, voir maintenant la contribution exhaustive et convaincante de Bowersock 2004, notamment p. 61-62, pour l'influence d'usages locaux sur la langue d'Artémidore.

70. II 55, P 184, 14-17 et 184, 22-185, 2.

71. Sur la détérioration de la situation de l'âme dans l'au-delà : HoHeisel 1993, p. 321-322.

72. Pour d'autres passages touchant la conception de la mort comme fin des incertitudes: BINDER 1991; WIEGELS 1991, p. 9-10.

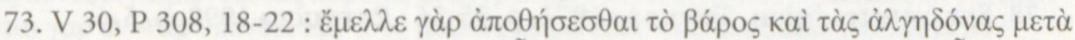

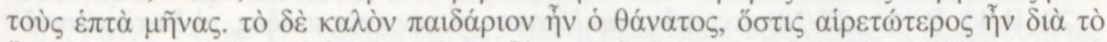

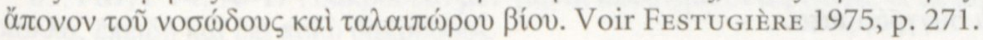


tion de la mort personnifiée emportant les vivants dans l'au-delà n'existait pas, à l'exception de la représentation, attestée chez Hésiode, de Thanatos et Hypnos comme fils de la Nuit ${ }^{74}$. La mort était toujours appréhendée à travers le cas concret des défunts et de leur trépas.

\section{LA MANIÈRE DONT LA MORT DÉTERMINE J.A VIE}

En divers passages, Artémidore atteste la conception selon laquelle la mort représentait le point d'aboutissement de la vie et son but. Ainsi lorsqu'il dit : « car les morts sont arrivés au dernier terme, comme ceux qui ont vaincu à ces Jeux », et "l'un et l'autre, et le mariage et la mort, sont pour les hommes des fins, et l'un indique toujours l'autre ${ }^{75}$ ". Le grec contient là un jeu de mots, dans la mesure où $\tau \dot{\lambda} \lambda \varsigma_{\zeta}$ signifie à la fois but et fin. Cela apparaît aussi lorsque des malades rêvent de courses de sprint et que l'interprétation renvoie à une mort imminente : ils atteignent en effet au but de la vie et terminent ainsi leur existence. Si donc la mort est le but et la fin de la vie et que celle-ci - comme nous l'avons montré - ne continue pas dans l'au-delà, alors se pose la question de savoir ce qui reste de la vie d'un homme. Sur ce sujet, on rencontre d'abord une affirmation lapidaire : « La mort rompt tous les liens ${ }^{76}$ ", c'est-à-dire que, même pour ceux qui restent, la séparation est insurmontable ${ }^{77}$. C'est pourquoi il est de la plus haute importance de laisser derrière soi une trace matérielle qui représente la mémoire du défunt ${ }^{78}$. De ce point de vue, selon Artémidore, le père de famille et l'homme de lettres sont particulièrement bien placés, car « ceux-ci laisseront comme monuments $(\mu \nu \eta \mu \varepsilon \tilde{\alpha} \alpha)$ d'eux-mêmes leurs enfants, et les autres laisseront comme

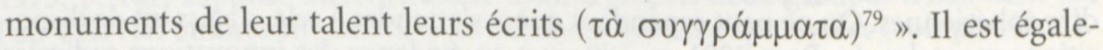

74. Hésiode, Théogonie, v. 212 : voir là-dessus et sur d'autres passages WöHrLE 1995, p. 19-23 ; Albinus 2000, p. 200-201 ; van Hooff 2005. Chez Hésiode, les rêves sont eux aussi fils de la Nuit, et donc frères d'Hypnos et Thanatos.

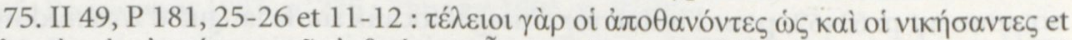

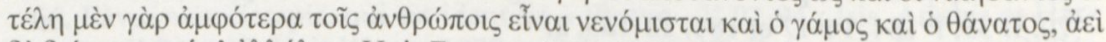

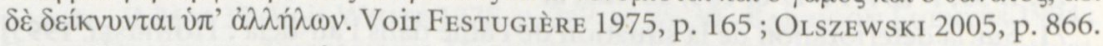

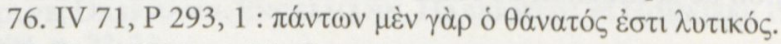

77. Veit 2008, p. 24. Gladigow 1980 souligne que les conceptions de l'au-delà étaient très largement déterminées par les besoins de ceux qui restaient en ce monde.

78. Sur cette tradition, Toyndee 1971, p. 35-36 ; Petrucci 1998, p. 5-6.

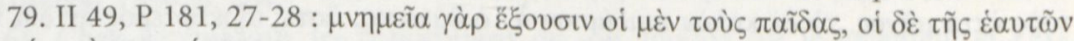

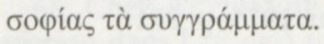


ment positif, pour quelqu'un qui est encore sans enfant, de rêver qu'il possède une tombe ou qu'il la fait construire, car il aura un enfant et le laissera derrière lui comme "monument ${ }^{80} "$. D'une manière générale, il n'est pas étonnant de trouver beaucoup de passages, dans les Oneirokritika d'Artémidore, où les rêves de célibataires sans enfants, qui sont du reste majoritairement de sexe masculin, sont discutés et interprétés comme annonçant un mariage et des enfants. C'est avec cet arrière-plan que l'on peut comprendre pourquoi le mariage était considéré comme un des buts de la vie et pourquoi les enfants étaient importants : dans l'oikos, dans la famille, ce n'est pas seulement la lignée qui se perpétue et le nom du défunt qui continue à être porté - nous rencontrons à nouveau la question de la prise en charge des parents par les enfants. Quant à la question de savoir si une mort imminente et annoncée par des rêves affectait concrètement le comportement des hommes, il n'est pas possible de le déterminer. La conscience générale de la mort devait sans aucun doute mettre en mouvement des stratégies rationnelles de comportement.

Il y a enfin les contrecoups émotionnels, dont relève en particulier le deuil $\left(\lambda \cup ́ \pi \eta^{81}\right)$. Celui-ci est bien présent chez Artémidore, et ce non seulement à travers les pleurs et les lamentations adressées au mort ou bien le deuil du père qui se consume comme dans un feu dans la douleur ( $\pi \varepsilon \dot{v} \theta 0 \varsigma$ ) d'avoir perdu un fils, mais aussi à travers des pratiques concrètes qui modifiaient pour un certain temps la vie quotidienne. Les gens en deuil se coupent les cheveux, car " c'est un vieil usage, en cas de deuil, de s'épiler les sourcils $[\ldots]$ », " et en effet dans les deuils, on se mutile les joues [...] », ou bien : "Les femmes en effet, dans le deuil, se mutilent aussi les seins ${ }^{82}$. " En outre, manger des oignons en rêve signifie la mort, " car ceux qui meurent versent peu de larmes, mais ceux qui sont endeuillés en versent beaucoup, puisqu'aussi bien ils pleurent longtemps ${ }^{83}$ ». Artémidore ne nous apprend rien sur la

80. Pour la signification du tombeau lui-même : CoRmack 2004, p. 147-160.

81. Remarques fondamentales à ce sujet chez Hopkins 1983, p. 121-127; Albinus 2000, p. 292.

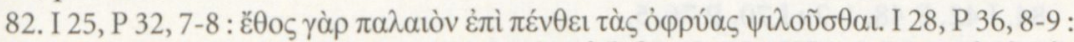

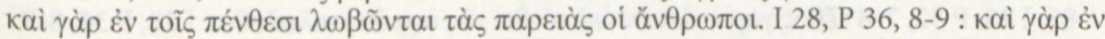

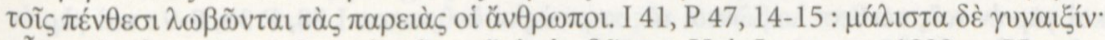

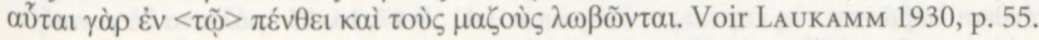

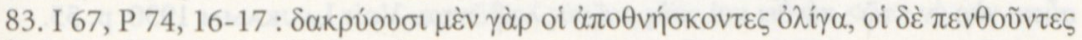

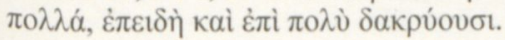


durée exacte du deuil ni sur la raison pour laquelle le rêve que le bras subit un dommage ou celui que l'on consomme de la viande de bœuf - à cause de son caractère coriace - annoncent le deuil ${ }^{84}$. Il n'est pas rare que le deuil soit présenté comme la conséquence d'un rềve, sans qu’un rapport explicite avec la mort soit posés5.

Une dernière chose encore : il serait pour nous extraordinairement captivant de connaître la réaction des hommes de ce temps lorsqu'un interprète des rêves leur annonçait leur mort et même, éventuellement, le moment où elle devait arriver. Rien n'est dit à ce sujet chez Artémidore, et ce point est de toute façon difficile à éclaircir dans la mesure où l'attitude à l'égard de la mort ainsi que les circonstances entourant chaque rêve étaient extrêmement variées.

\section{ConcLusion}

Le thème de la mort présente chez Artémidore bien des facettes, dont certaines, essentielles, ont pu être discutées ; d'autres, comme par exemple la question de la signification des édifices funéraires en eux-mêmes ${ }^{86}$, doivent être mises en réserve pour des recherches futures. Sur le plan des décors du rêve, nous avons fait connaissance de divers symboles qui relevaient avant tout d'une mythologie extraordinairement vivante et tout simplement omniprésente ainsi que des différents types de mort. Tant sur le plan symbolique que chez les rêveurs, le contexte privilégié est celui des membres de la famille et des malades : les uns en raison d'une conception matérialiste de la vie selon laquelle la perpétuation de la famille et la prise en charge des parents par les enfants étaient des points essentiels; les autres en raison de leur proximité à la fois réelle et mentale avec la mort. Ensuite, la mort a été envisagée comme une frontière stricte et insurmontable, une frontière dont le franchissement - ne serait-ce qu'en rêve - doit être empêché en toutes circonstances et cela en raison des conséquences funestes qu'il entraînait. C'est à cela que servaient notamment les divers rites que l'on devait accomplir autour du cadavre et dans le cadre des funérailles ou du deuil. Ce qu'il

\section{I 42, P 48, 1-2; I 70, P 76, 5.}

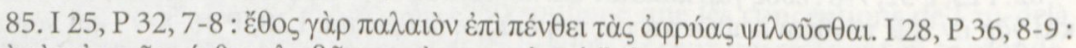

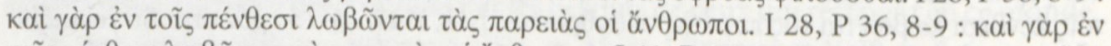

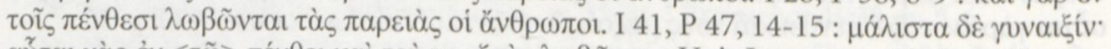

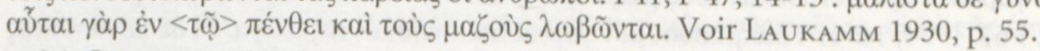

86. OlszeWSKI 2005, p. 867 et 873. 
y a de fondamental pour la conception de l'au-delà est la séparation qui s'opère dans le défunt entre le corps, qui revient à la terre, et l'âme qui descend dans l'Hadès ou qui monte au ciel. Pour quelles raisons et dans quels cas s'applique l'une ou l'autre de ces translations - cela n'est pas dit. Artémidore montre du reste une certaine réticence, voire du scepticisme, envers la seconde de ces conceptions. Il prend cependant en compte les circonstances et conditions d'existence propres à chaque individu dans la mesure où la mort et même l'absence de toute émotion pour l'âme dans l'Hadès pouvaient être interprétés comme une libération à l'égard des souffrances. Même les émotions ressenties face à la mort jouaient dans l'existence un rôle significatif. Mais, parce qu'il n'y avait pas après la mort de vie active dans l'au-delà, les efforts des hommes dans la vie se concentraient sur les différentes formes de mémoire.

Gregor Weber Université d'Augsbourg 